\title{
An Edge Quadtree for External Memory
}

\author{
Herman Haverkort $^{1}$ and Mark McGranaghan ${ }^{2 \star}$ and Laura Toma ${ }^{2 \star \star}$ \\ 1 Eindhoven University of Technology, the Netherlands \\ 2 Bowdoin College, USA
}

\begin{abstract}
We consider the problem of building a quadtree subdivision for a set $\mathcal{E}$ of $n$ non-intersecting edges in the plane. Our approach is to first build a quadtree on the vertices corresponding to the endpoints of the edges, and then compute the intersections between $\mathcal{E}$ and the cells in the subdivision. For any $k \geq 1$, we call a K-quadtree a linear compressed quadtree that has $O(n / k)$ cells with $O(k)$ vertices each, where each cell stores the edges intersecting the cell. We show how to build a K-quadtree in $O(\operatorname{sort}(n+l))$ I/O's, where $l=O\left(n^{2} / k\right)$ is the number of such intersections. The value of $k$ can be chosen to trade off between the number of cells and the size of a cell in the quadtree. We give an empirical evaluation in external memory on triangulated terrains and USA TIGER data. As an application, we consider the problem of map overlay, or finding the pairwise intersections between two sets of edges. Our findings confirm that the K-quadtree is viable for these types of data and its construction is scalable to hundreds of millions of edges.
\end{abstract}

\section{Introduction}

The word quadtree describes a class of data structures that partition the space hierarchically and are defined by a stopping criterion that decides when a region is not subdivided further. In $2 \mathrm{D}$, the quadtree recursively divides a square containing the data into four equal regions (quadrants or cells), until each region satisfies the stopping condition (usually, when a cell is "small" enough). The set of cells that are not split further define the leaves of the tree and represent a subdivision of the input region. Quadtrees have been used for many types of data (points, line segments, polygons, rectangles, curves) and many types of applications. For an ample survey we refer to [12].

In this paper we are interested in quadtrees for data sets that are so large that they do not fit in the internal memory of the computer, so that at any time, most of the data has to reside in external memory. To analyze the efficiency of the construction and query algorithms in this case, we use the standard I/Omodel by Aggarwal and Vitter [2]. In this model, a computer has an internal memory of size $M$ and an arbitrarily large disk. The data is stored on disk in blocks of size $B$, and, whenever the algorithms needs to access data not present in memory, it loads the block(s) containing the data from disk. The I/o-complexity

\footnotetext{
* Supported by Bowdoin Freedman Fellowship and NSF award no. 0728780.

** Supported by NSF award no. 0728780.
} 
of an algorithm is the number of I/O's it performs, that is, the number of blocks transferred (read or written) between main memory and disk. Sorting takes $\operatorname{sort}(n)=\Theta\left(\frac{n}{B} \log _{M / B} \frac{n}{B}\right)$ I/O's [2]; scanning takes $\operatorname{scan}(n)=\Theta(n / B)$ I/O's.

Quadtrees can be viewed as trees representing the hierarchical space decomposition, or as the set of leaf cells ordered along a space-filling curve. The latter variant of quadtree, called the linear quadtree, was introduced by Gargantini [5]. The linear quadtree is particularly useful when dealing with disk-based structures, because its space requirements are smaller. Quadtrees are known to perform well empirically in many different applications, but their worst-case behaviour is not ideal, except in the simplest cases. Given a set of $n$ points in the plane, a quadtree that splits a region until it contains at most one point can have unbounded size. However, it is known how to construct a compressed quadtree of $O(n)$ cells which each have at most one point. In a compressed quadtree, paths consisting of nodes with only one non-empty child are replaced by a single node, with all empty children merged into one. Throughout this paper, the concept of quadtrees will encompass both compressed and uncompressed quadtrees.

Building a quadtree on a set of $n$ non-intersecting edges in the plane, rather than points, is harder. We refer to a quadtree for a set of edges as an edge quadtree, and we denote by $l$ the number of intersections between the edges and the cells in the quadtree subdivision. One way to build an edge quadtree is to first build a compressed quadtree on the endpoints of the edges, and then compute the intersections between the edges and the cells in the subdivision. In the worst case, each edge can intersect almost all cells, giving a quadtree of quadratic size. Another type of edge quadtree may split a region until it intersects a single edge. Since the distance between two edges can be arbitrarily small, the resulting quadtree has unbounded size. Other edge quadtrees can be defined by formulating specific stopping criteria. Such structures were described by Samet et al. [14, 13,10]. The PM quadtree [14] allows a region to contain more than one edge if the edges meet at a vertex inside the region. Variants of PM quadtrees differ in how to handle regions that contain no vertices. The segment quadtree [13] is a linear quadtree in which a leaf cell is either empty, contains one edge and no vertices, or contains precisely one vertex and its incident edges. The PMR quadtree [10] is a linear quadtree where each region may have a variable number of segments and regions are split if they contain more than a predetermined threshold. Hoel and Samet [9] compared the PMR quadtree with some variants of R-trees on TIGER data, in terms of storage requirements, construction time (disk I/O's), and a number of queries. They find that the PMR quadtree performs well compared to the R-tree for map overlay. Subsequently, improved algorithms for the construction of the PMR quadtree have been proposed $[7,6,8]$. The algorithms perform well in practice, but there are several disadvantages: First, the stopping rule of the PMR quadtree means that the size of a leaf depends on both the splitting threshold and the depth of the leaf, and the quadtree depends on the insertion order. Second, the complexity is analysed in terms of various parameters that depend on the data, in a way that is not well understood. Finally, the algorithms are fairly complex, and the performance is not worst-case optimal. 
Quadtrees in the I/O-model were described by Agarwal et al. [1] and De Berg et al. [4]. Agarwal et al. describe an algorithm for constructing a quadtree on a set of $n$ vertices in the plane such that each cell contains $O(k)$ vertices (for any $k \geq 1$ ), that runs in $O\left(\frac{n}{B} \frac{h}{\log M / B}\right)$ I/O's, where $h$ is the height of the quadtree. This is $O(\operatorname{sort}(n))$ I/O's when $h=O(\log n)$ i.e. the vertices are nicely distributed. Their algorithm was implemented and tested in practice as part of an application to interpolate LIDAR datasets into grids. De Berg et al. described the star-quadtree for triangulations, and the guard-quadtree for sets of edges in the plane, which contain at most one vertex per cell and can be constructed in $O(\operatorname{sort}(n+l))$ I/O's, where $l=O\left(n^{2}\right)$ is the number of edge-cell intersections. The star- and guard-quadtrees are designed to exploit fatness and density: for fat triangulations ${ }^{3}$ and sets of edges of low density ${ }^{4}$, respectively, the star-quadtree and guard-quadtree have the property that each cell intersects $O(1)$ edges, thus $l=O(n)$. An experimental evaluation of these structures has not been reported.

Our Contribution. We consider building an edge quadtree for a set $\mathcal{E}$ of $n$ non-intersecting edges. Let $k \geq 1$ be a user defined parameter. Our algorithm has two steps: First it builds, in $O(\operatorname{sort}(n))$ I/O's, a compressed linear quadtree on the endpoints of $\mathcal{E}$ with $O(n / k)$ cells in total and such that each cell has $O(k)$ vertices. Second, it computes the intersections between the edges and the quadtree subdivision in $O(\operatorname{sort}(n+l))$ I/O's (where $l=O\left(n^{2} / k\right)$ is the total number of intersections). We refer to the resulting quadtree as a K-quadtree.

The first step, constructing the quadtree subdivision, is a generalization of the algorithm for building guard-quadtrees in [4]. Compared to the algorithm by Agarwal et al. [1], our algorithm has better complexity, is much simpler, and gives an upper bound on the number of cells in the subdivision. The second step, which we refer to as edge distribution, is based on an idea communicated to us by Doron Nussbaum. For $k=1$ the algorithm has the same complexity as in [4], but it is simpler and faster.

In Section 4 we give an empirical evaluation of K-quadtrees on triangulated terrains (in GIS: TINs) and USA TIGER data. We examine the size of the quadtree, the size of a cell and the construction time for different values of $k$. We use test datasets up to 427 million edges, two orders of magnitude larger than in related work $[7,6,8]$. On TINs and TIGER data the K-quadtrees have linear size, which matches the results of $[9,7,6,8]$. In terms of construction time (or bulk loading), a comparison with previous work is difficult. The running times in [9] are given in terms of disk block accesses, not the total execution time. The tests in $[7,6,8]$ are performed on three TIGER data sets, the largest one having approx. 200,000 edges, on a machine with 64MB RAM. Our largest TIGER bundle has 427 million edges (6.8 GB), and we use machines with 512MB RAM. Furthermore a precise comparison is not possible without knowing all the tuning parameters used in [8].

\footnotetext{
${ }^{3}$ A triangulation such that every angle is larger than some fixed positive constant $\delta$

${ }^{4}$ Any disk $D$ is intersected by at most $\lambda$ edges whose length is at least the diameter of $D$, for some fixed constant $\lambda$.
} 
As an application of quadtrees we consider one of the basic operations in GIS and spatial data structures, map overlay: computing the pairwise segment intersections (overlay) between two sets of edges. Given two sets of edges, each pre-processed as a quadtree, their intersections can be computed in a very simple manner by scanning the two quadtrees as in [4]. We implemented map overlay and report on the running time using various values of $k$.

Overall, our experimental results confirm that the K-quadtree is viable for very large TIN and TIGER data. These represent relatively simple classes of inputs; however they arise frequently in practice and have been used extensively as tests beds for spatial index structures. Further experiments are necessary for other types of data, and we leave this as a topic for future work.

\section{Preliminaries}

For simplicity, we assume that the edges $\mathcal{E}$ lie in the unit square. For quadtree background and notation see e.g. [11,4]. A square that is obtained by recursively dividing the input square into quadrants is called a canonical square. To order the quadrants, we use the Z-order space-filling curve that visits the 4 quadrants, recursively, in order SW, NW, SE, NE. Z-order gives a well-defined ordering between the cells in the quadtree subdivision, as well as between any two points. For a point $p=\left(p_{x}, p_{y}\right)$ in the unit square, define its Z-index $Z(p)$ to be the value in the range $[0,1)$ obtained by interleaving the bits in the fractional parts of $p_{x}$ and $p_{y}$. The value $Z(p)$ is sometimes called the Morton block index of $p$. The $\mathrm{Z}$-order of two points is the order of their z-indices. The Z-indices of all points in a canonical square $\sigma$ form an interval $\left[z_{1}, z_{2}\right)$ of $[0,1)$, where $z_{1}$ is the Z-index of the bottom left corner of $\sigma$. A compressed quadtree subdivision has two types of cells: canonical squares, and donut cells, corresponding to empty nodes that were merged together. A donut cell is the difference between two canonical squares $\left[z_{1}, z_{2}\right]-\left[z_{3}, z_{4}\right]$ and is represented as the union of two intervals $\left[z_{1}, z_{3}\right] \cup\left[z_{4}, z_{2}\right]$.

With this notation, a (compressed) quadtree subdivision corresponds to a subdivision $\mathcal{Q}$ of the Z-order curve, and it can be viewed as a set of consecutive, adjacent, non-overlapping intervals, covering $[0,1)$, in Z-order: $\mathcal{Q}=\left\{\left[z_{1}=\right.\right.$ $\left.\left.0, z_{2}\right),\left[z_{2}, z_{3}\right),\left[z_{3}, z_{4}\right), \ldots\right\}$; Each interval corresponds to a cell $\sigma_{i}$, which is either a canonical square or a part of a donut. We represent a K-quadtree as a subdivision of the Z-order curve where each intersection of an edge $e$ with a cell $\sigma$ corresponding to the interval $\left[z_{1}, z_{2}\right)$ is represented by storing edge $e$ with key $z_{1}$. A K-quadtree is thus a list of pairs $\left\{\left(z_{1}, e\right)\right\}$, stored in order of $z_{1}$.

In the rest of the paper we denote by $l$ the number of intersections between $\mathcal{E}$ and the cells in the quadtree subdivision, and we use the terms quadtree, quadtree subdivision and subdivision interchangeably.

\section{Constructing a K-quadtree}

In this section we describe our algorithm for building a K-quadtree. Let $k \geq 1$ be a user defined parameter. Our algorithm has two steps: In the first step 
it ignores the edges and builds, in $O(\operatorname{sort}(n))$ I/O's, a linear quadtree on the endpoints of the edges. The quadtree has $O(n / k)$ cells in total, each containing $O(k)$ vertices. Second, it computes the intersections between the edges and the quadtree subdivision in $O(\operatorname{sort}(n+l))$ I/O's. We describe the two steps below.

Constructing the subdivision. Let $\mathcal{P}=\left\{p_{0}, p_{1}, p_{2}, \ldots\right\}$ be the vertices of $\mathcal{E}$. A straightforward idea to build a quadtree with $O(k)$ vertices per cell would be to start with one of the standard algorithms for building a quadtree with at most one vertex per cell, and then traverse the subdivision and merge cells into cells of size $O(k)$. However, we would like to avoid generating first a larger subdivision and then merging its cells to get a smaller subdivision. Another approach might be to build the quadtree top-down: stop if the cell contains $O(k)$ vertices, otherwise split the cell and distribute the points among the four children, and continue on the children recursively; however, this may take $\Theta\left(n^{2}\right)$ time as the quadtree may have height $\Theta(n)$.

Our idea to generate a quadtree subdivision with $O(n / k)$ cells and $O(k)$ vertices in each cell directly, is a simple and elegant generalization of an algorithm in [4]. Assume that $\mathcal{P}$ has been sorted in Z-order, and denote $\mathcal{P}_{k}$ the set of every $k^{\text {th }}$ point in $\mathcal{P}: \mathcal{P}_{k}=\left\{p_{0}, p_{k}, p_{2 k}, \ldots\right\} \subset \mathcal{P}$. The idea is to build the quadtree subdivision induced by $\mathcal{P}_{k}$ : for every pair of consecutive points in $\mathcal{P}_{k}$, we find their smallest enclosing canonical square, and output the Z-indices corresponding to the $4 \mathrm{z}$-intervals of the quadrants of this square. We claim that:

Lemma 1. The resulting list of $\mathrm{Z}$-indices represents a compressed quadtree subdivision with $O(n / k)$ cells and $O(k)$ vertices per cell.

Proof. Every pair of consecutive points of $\mathcal{P}_{k}$ causes a split, and generates 4 cells, therefore $O(n / k)$ cells; each cell contains at most one point of $\mathcal{P}_{k}$ inside (or otherwise it would have been split), therefore $O(k)$ points of $\mathcal{P}$.

Assuming that the operations involving z-indices take $O(1)$ time, this step runs in $O(n)$ time and $O(\operatorname{scan}(n))$ I/O's. With the help of the stack described in the appendix of [4], we can actually output the z-indices in increasing order without additional I/O. Thus we get a compressed quadtree subdivision represented by a list of Z-intervals, in Z-order of their first endpoint: $\mathcal{Q}=\left\{\left[z_{1}=\right.\right.$ $\left.\left.0, z_{2}\right],\left[z_{2}, z_{3}\right], \ldots\right\}=\left\{I_{1}, I_{2}, \ldots\right\}$. We note that in practice we represent the second endpoint of the intervals implictly.

An algorithm for edge distribution when $k=1$. Let $\mathcal{Q}=\left\{I_{1}, I_{2}, \ldots\right\}$ be a subdivision of the endpoints of $\mathcal{E}$ obtained by the algorithm described above, and assume $\mathcal{Q}$ is given in Z-order. We will now first consider the case $k=1$, i.e. every cell contains at most one vertex, and the total number of cells is $O(n)$. We describe how to find the intersections between $\mathcal{Q}$ and $\mathcal{E}$ in $O(\operatorname{sort}(n+l))$ I/O's. Later we will show how to generalize this process to a subdivision with $O(k)$ vertices in a cell, where $k>1$.

We assume edges are oriented from left to right, vertical segments are oriented upwards, and let $\mathcal{E}_{+}$and $\mathcal{E}_{-}$denote the edges of positive and negative slope, respectively. The crux of the algorithm is to process the edges of positive and negative slope separately. We describe below the two steps. 

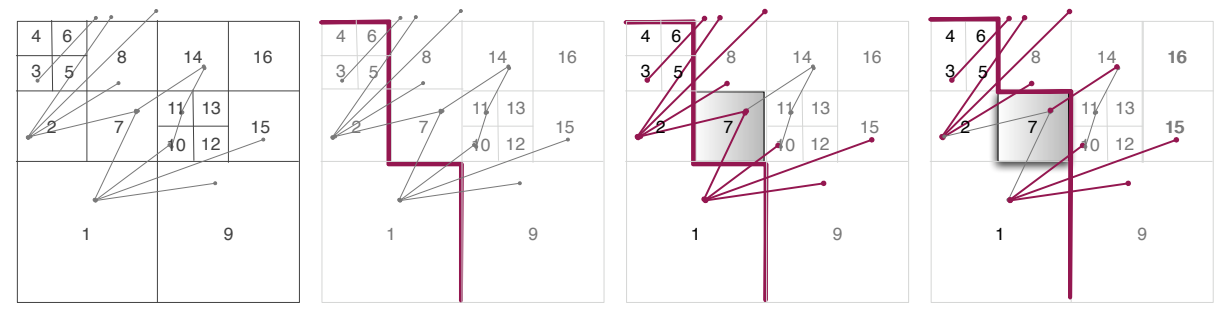

Fig. 1. (a) $\mathcal{E}_{+}$(b) $B_{7}$ (c) $X_{7}$ and processing cell $\sigma_{7}$ (d) $X_{8}$

Distributing the edges of positive slope. The idea is to scan $\mathcal{Q}$, one interval at a time, and find all the edges in $\mathcal{E}_{+}$intersecting the cell corresponding to the current interval. Let $I_{j}=\left[z_{j}, z_{j+1}\right]$ be the next interval we read from $\mathcal{Q}$, and let $\sigma_{j}$ be the corresponding cell in the subdivision. There are two types of intersections between $\sigma_{j}$ and $\mathcal{E}_{+}$, see Fig. 1:

- First, there may be edges that intersect $\sigma_{j}$ and originate in $\sigma_{j}$;

- Second, there may be edges that intersect $\sigma_{j}$ and originate outside $\sigma_{j}$.

Intersections of the first type can be detected by scanning $\mathcal{Q}$ and $\mathcal{E}_{+}$in sync, as follows. Let $\mathcal{E}_{+}$be sorted in Z-order of the first endpoints of the edges. Let $I_{j}$ be the current interval in $\mathcal{Q}$, and let $e=(p, q)$ be the next edge in $\mathcal{E}_{+}$. To check whether $e$ originates in $\sigma_{j}$ means checking if $z(p) \in I_{j}$. This leads to the following algorithm: For each interval $I_{j} \in \mathcal{Q}$, we read from $\mathcal{E}_{+}$all the edges that originate in $\sigma_{j}$, and stop when encountering the first edge $e^{\prime}=\left(p^{\prime}, q^{\prime}\right)$ with $z\left(p^{\prime}\right)>z_{j+1}$. Then we continue with the next interval from $\mathcal{Q}$, in the same fashion. Because the edges in $\mathcal{E}_{+}$are stored in Z-order of their first endpoint, we know that once we encounter an edge with $z\left(p^{\prime}\right)>z_{j+1}$, then all subsequent edges have the same property and none of them can originate in $\sigma_{j}$. This runs in $O\left(\operatorname{scan}\left(|\mathcal{Q}|+\left|\mathcal{E}_{+}\right|\right)\right)=O(\operatorname{scan}(n))$ I/O's.

The harder problem is finding the intersections of $\sigma_{j}$ with the edges that originate outside $\sigma_{j}$. It is here that we exploit that $\mathcal{E}_{+}$and $\mathcal{E}_{-}$are processed separately. The key observation is that any edge of positive slope that intersects $\sigma_{j}$ originates in a cell that comes before $\sigma_{j}$, in Z-order. In general we have:

Lemma 2. An edge of positive slope intersects the cells in $\mathcal{Q}$ in Z-order.

Consider the current interval $I_{j}$ in $\mathcal{Q}$. By Lemma 2 it follows that all the edges that intersect $\sigma_{j}$ and do not start in $\sigma_{j}$ must originate in a cell $\sigma_{i}$ before $\sigma_{j}$, that is $i<j$. Let $B_{j}$ denote the boundary between the cells explored before $\sigma_{j}, \bigcup_{i<j} \sigma_{i}$ and the rest of the cells $\bigcup_{i \geq j} \sigma_{i}$, for any $j \geq 1$. The edges in $\mathcal{E}_{+}$that originate (but do not end) in a cell before $\sigma_{j}$ will intersect the boundary $B_{j}$; let $X_{j}$ be the set of these edges. See Fig. 1. More precisely, let $X L_{j}$ be the edges of $X_{j}$ that intersect $B_{j}$ between the left edge of the unit square and the lower left corner of $\sigma_{j}$, and let $X B_{j}$ be the edges of $X_{j}$ that intersect $B_{j}$ between the lower left corner of $\sigma_{j}$ and the bottom edge of the unit square. Here, if $\sigma_{j}$ is the 
second part of a donut, we define its lower left corner as the upper right corner of the hole, which is, in fact, the upper right corner of $\sigma_{j-1}$.

Lemma 3. $B_{j}$ is a monotone staircase and the intersection of $\sigma_{j}$ and $B_{j}$ covers a connected part of $B_{j}$.

The algorithm will maintain $X_{j}$ on two stacks $S L$ and $S B$, keeping the following invariant: before processing an interval $I_{j}$ from $\mathcal{Q}$, the stack $S L$ contains, from bottom to top, the edges of $X L_{j}$ in the order of their intersections with $B_{j}$ from the left edge of the unit square to the lower left corner of $\sigma_{j}$; the stack $S B$ contains, from bottom to top, the edges of $X B_{j}$ in the order of their intersections with $B_{j}$ from the bottom edge of the unit square to the lower left corner of $\sigma_{j}$. Initially, for $j=1$, the boundary $B_{1}$ is empty and both stacks are empty.

The algorithm now scans $\mathcal{Q}$ and $\mathcal{E}_{+}$. When $I_{j}$ is the next interval in $\mathcal{Q}$, the algorithm reads all edges that originate in $\sigma_{j}$ from $\mathcal{E}_{+}$, and pops all edges that intersect $\sigma_{j}$ "from before" from $S L$ and $S B$. Out of these edges, we push those that leave $\sigma_{j}$ between the upper left and upper right corner onto $S L$, and those that leave $\sigma_{j}$ between the lower right and the upper right corner onto $S B$, in order of their intersections with the boundary of $\sigma_{j}$ towards the upper right corner (if $\sigma_{j}$ is part of a donut surrounding $\sigma_{j+1}$, we take the lower left corner of $\sigma_{j+1}$ as the upper right corner of $\sigma_{j}$ ). Finally we establish the invariant for the next interval: if the lower left corner of $\sigma_{j+1}$ lies above the lower left corner of $\sigma_{j}$, we do this by popping edges from $S L$ and pushing them onto $S B$ one by one until $S L$ is empty or the top of $S L$ intersects $B_{j+1}$ between the left edge of the unit square and the lower left corner of $\sigma_{j+1}$; otherwise we establish the invariant in a symmetric way by moving edges from $S B$ to $S L$.

From Lemma 3 and the invariant it follows that before processing cell $\sigma_{j}$, all edges of $X_{j}$ that intersect $\sigma_{j}$ are on top of $S L$ or $S B$, and thus the algorithm correctly finds all edges intersecting $\sigma_{j}$ and correctly restores the invariant after every step. It remains to analyse the efficiency of the algorithm. We claim that:

Lemma 4. Each edge of $\mathcal{E}_{+}$is pushed onto $S B$ at most once and pushed onto $S L$ at most once for each intersection with $\mathcal{Q}$.

For a brief justification, consider a square and its four quadrants. Let $h$ be the left half of the horizontal midline of the square, and let $H$ be the edges intersecting $h$. These edges leave the lower left quadrant across its top edge and are therefore pushed onto $S L$; they are moved to $S B$ just before processing the first cell in the upper left quadrant. From there, the edges of $H$ will never move back to $S L$ while still representing the intersection with $h$, as this would only happen if the lower left corner of the next cell $\sigma_{j+1}$ is to the right of the intersections of $H$ with $h$. However, by the monotonicity of $B_{j+1}$, this can only happen after all cells that touch $h$ from above and to the left of $\sigma_{j+1}$ have already been processed, at which time the edges of $H$ must have been removed from the stack. Similarly, the edges crossing the vertical midline of the square leave the quadrants on the left across their right edges and are therefore pushed onto $S B$; they are moved to $S L$ just before processing the first cell in the lower 
right quadrant. From there they are removed as we traverse the leftmost cells within the quadrants on the right from bottom to top.

Let $l^{+}$be the number of intersections between $\mathcal{E}_{+}$and $\mathcal{Q}$. Putting everything together it follows that the intersections of $\mathcal{E}_{+}$and $\mathcal{Q}$ can be found in $O(\operatorname{scan}(n+$ $\left.l^{+}\right)$) I/O's once $\mathcal{E}_{+}$and $\mathcal{Q}$ are sorted.

Distributing the edges of negative slope. To distribute the edges of negative slope, we observe that Lemma 2 holds for edges of negative slope if we consider a different Z-order: $\mathrm{Z}^{\prime}=\mathrm{NW}, \mathrm{NE}, \mathrm{SW}, \mathrm{SE}$. We convert $\mathcal{Q}$ to a subdivision $\mathcal{Q}^{\prime}$ onto the Z'-order curve, find the intersections with $\mathcal{E}_{-}$using the same algorithm as above, and map the intersections back to the cells in $\mathcal{Q}$. All these steps run in $O\left(\operatorname{sort}\left(n+l^{-}\right)\right)$I/O's, where $l^{-}$stands for the number of intersections between $\mathcal{E}_{-}$and $\mathcal{Q}$. Overall, the intersections between $\mathcal{Q}, \mathcal{E}_{+}$and $\mathcal{E}_{-}$can be found in $O(\operatorname{sort}(n+l))$ I/O's, where $l=l^{+}+l^{-}$is the total number of intersections.

Distributing edges in a K-quadtree Above we described how to find the intersections between $\mathcal{E}$ and a quadtree subdivision where each cell contains at most one vertex $(k=1)$. We now describe briefly how to extend the algorithm to $k>1$.

Recall that the algorithm for $k=1$ reads intervals in order from $\mathcal{Q}$ while maintaining the stacks $S L$ and $S B$. For each interval $I_{j}$ it: (a) finds the edges that originate in $\sigma_{j}$; (b) finds the edges that intersect $\sigma_{j}$ and originate outside $\sigma_{j}$; (c) merges these two groups of edges in order onto the stacks. The only step that is different when $k>1$ is (c). In this case the edges that originate in $\sigma_{j}$ need to be carefully interleaved with the edges of $X_{j}$. Note that we read the edges originating in $\sigma_{j}$ from $\mathcal{E}_{+}$in Z-order of their start point, which is not necessarily the order in which they will appear in $X_{j+1}$. For each edge we find the intersection with $\sigma_{j}$, and then sort all edges intersecting $\sigma_{j}$ (the edges found on the stacks and the edges originating in $\sigma_{j}$ ) by the point where they leave $\sigma_{j}$. Since the boundary of $\sigma_{j}$ is a monotone staircase, sorting the edges by these exit points gives them in the order in which they appear on $B_{j+1}$. Overall the algorithm runs in $O(\operatorname{sort}(n+l))$ I/O's.

\section{Experimental results}

In this section we present an empirical evaluation of K-quadtrees on two types of data commonly used in GIS applications, triangulated terrains (TINs) and TIGER data. We implemented the construction algorithm described in Section 3 and experimented with various

values of $k$. The current implementation assumes that $k=O(M)$ and the number of edges that intersect a cell fit in memory; they are sorted using system qsort. We compare the resulting subdivisions in terms of total number of edge intersections, average number of edge intersections per cell, maximum number of intersections per cell, and construction time. For comparison we also implemented the construction algorithm in [4], denote QDT-1-OLD. As an application we consider the time to compute the pairwise segment intersections (overlay) 
between two sets of edges, which is one of the standard operations in GIS and spatial databases. Given two sets of edges, each pre-processed as a K-quadtree, their intersections can be computed in a very simple and efficient manner, while scanning the two quadtrees, see e.g. [4].

Let $e$ denote the number of edges in the input dataset, $c$ the number of cells in the quadtree subdivision, and $l$ the number of edge-cell intersections in the quadtree subdivision. For each quadtree we measured the following average quantities: (i) the average number of cells per input edge, $c / e$; (ii) the average number of edge-cell intersections per edge, $l / e$ (indicates the total size of the quadtree, relative to the input size); (iii) the average number of edges intersecting a cell, $l / c$ (indicates the average size of a cell in the quadtree).

Datasets. In the first set of experiments we built quadtrees on triangulated terrains, for which we ignored the elevation, with size up to $53.9 \cdot 10^{6}$ edges. The datasets represent Delaunay triangulations of elevation samples of real terrains. They have not been filtered to eliminate narrow triangles. For all our test datasets, the minimum angle is on the order of $0.001^{\circ}$ and the maximum angle close to $180^{\circ} ; 5 \%$ of the angles are below $18^{\circ}$ and $5 \%$ above $108^{\circ}$; the average minimum angle is around $33^{\circ}$; and the median angle $57^{\circ}$. The maximum number of edges incident on a vertex varies widely across all datasets, ranging between 31 and 356; the average incidence across all datasets is approx. 6. (Fig. 4(a)).

In the second set of experiments we used USA TIGER2006SE data. This consists of 50 datasets, one for each state, containing the roads, railways, boundaries and hydrography in the state. The size of a dataset ranges from 115,626 edges (DE), to 40.4 million edges (TX). We assembled 4 (larger) datasets: New England (25.8 million edges), East Coast (113.0 million edges), Eastern Half (208.3 million edges) and All US (427.7 million edges). (Fig 4(b)).

Platform. The algorithms are implemented in $\mathrm{C}$ and compiled with $\mathrm{g}++4.1 .2$ with optimization level -O3. All experiments were run on HP 220 blade servers, with an Intel $2.83 \mathrm{GHz}$ processor, $512 \mathrm{MB}$ of RAM and a $5400 \mathrm{rpm}$ SATA hard drive. The hard disk is standard speed for laptop hard-drives. As I/O-library we used IOStreams [15], an I/O-kernel derived from TPIE [3]. The only components used were scanning and sorting, so other I/O-libraries can be plugged in.

Results on triangulations. In the first set of experiments we computed K-quadtrees on TIN data for various values of $k \geq 1$, denoted QDT-K. The results are shown in Fig 2 and Fig. 5. Our construction algorithm is significantly faster than QDT1-OLD (210 minutes vs. 1071 minutes on a TIN with $\left.e=54 \cdot 10^{6}\right)$. As expected, when $k$ increases, the construction time decreases (Fig 2(a)); the number of cells in the quadtree decreases (Fig 2(b)) and the overall size of the quadtree decreases (since fewer cells lead to fewer edge-cell intersections, Fig 2(c)). On the other hand the average number of edge intersections per cell, $l / c$, increases (Fig 2(d)). For example, on a TIN with $e=54 \cdot 10^{6}$, QDT- 1 is built in 210 minutes, has $c=.6 e, l=2.9 e$ and $l / c=4.8$; QDT-100 is built in 57 minutes, and has $c=.004 e, l=1.2 e$ and $l / c=257$. Note that $l / c$ represents an average quantity 

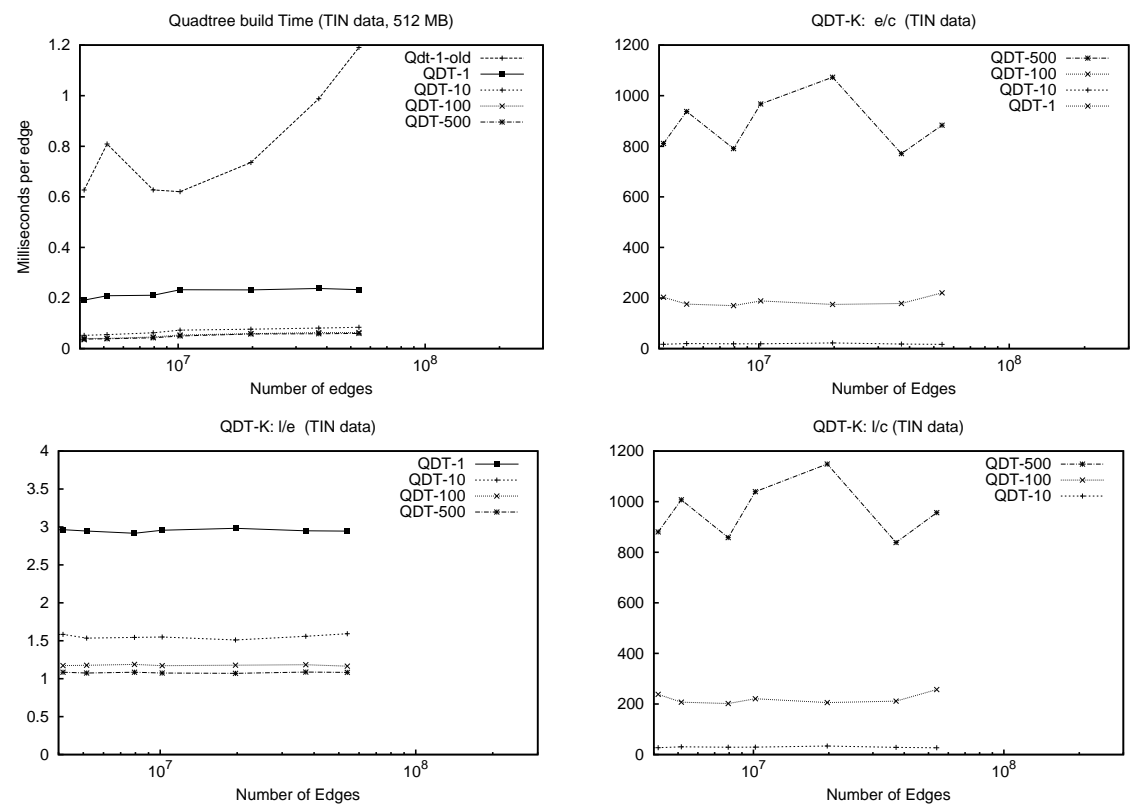

Fig. 2. Quadtree build times and sizes on TIN data (512MB RAM).

over the entire TIN and the maximum number of edges per cell can be much higher. In summary, for increasing $k$, QDT-K is built faster, has smaller overall size and larger cell size. Table 1 shows the various quadtree sizes and build times for one of the test TINs. The total size of the quadtree stays consistently small across all TINs, and appears to grow linearly with the number of edges.

Results on TIGER data. In the second set of experiments we computed Kquadtrees for TIGER data. The results are shown in Fig. 3. Same as for TINs, the build time gets faster up to $k=100$, and then levels. E.g., on EastHalf $\left(e=208 \cdot 10^{6}\right)$, it takes 24.7 hours to build QDT-1, 9.0h to build QDT-10, 4.8h to build QDT-100, and 4.5h to build QDT-500; on AllUSA $\left(e=428 \cdot 10^{6}\right)$, QDT-100 can be built in 9.7h. The algorithms run at $70 \% \mathrm{CPU}$ utilization. Similar to $[7,6,8]$, we found that the bottleneck in quadtree construction is edge distribution; in our case it accounts for more than $90 \%$ of the total running time, and runs at more than $70 \%$ CPU. Even with our new algorithm, building a QDT-1 is practically infeasible on moderately large data, taking more than $20 \mathrm{~h}$.

The average quadtrees sizes are relatively consistent across all datasets, which is somewhat surprising. QDT-1 has one edge per cell $(l / c=1)$ on average and an overall size $l=3 e$. We also computed the maximum cell size (Fig. 3(c)), which varies widely from state to state; for example, the largest cell in the EastHalf bundle intersects 58 edges, while for states like ME and VT, the largest cell intersects 8 edges. For increasing values of $k$, QDT-K has a larger average cell, but 

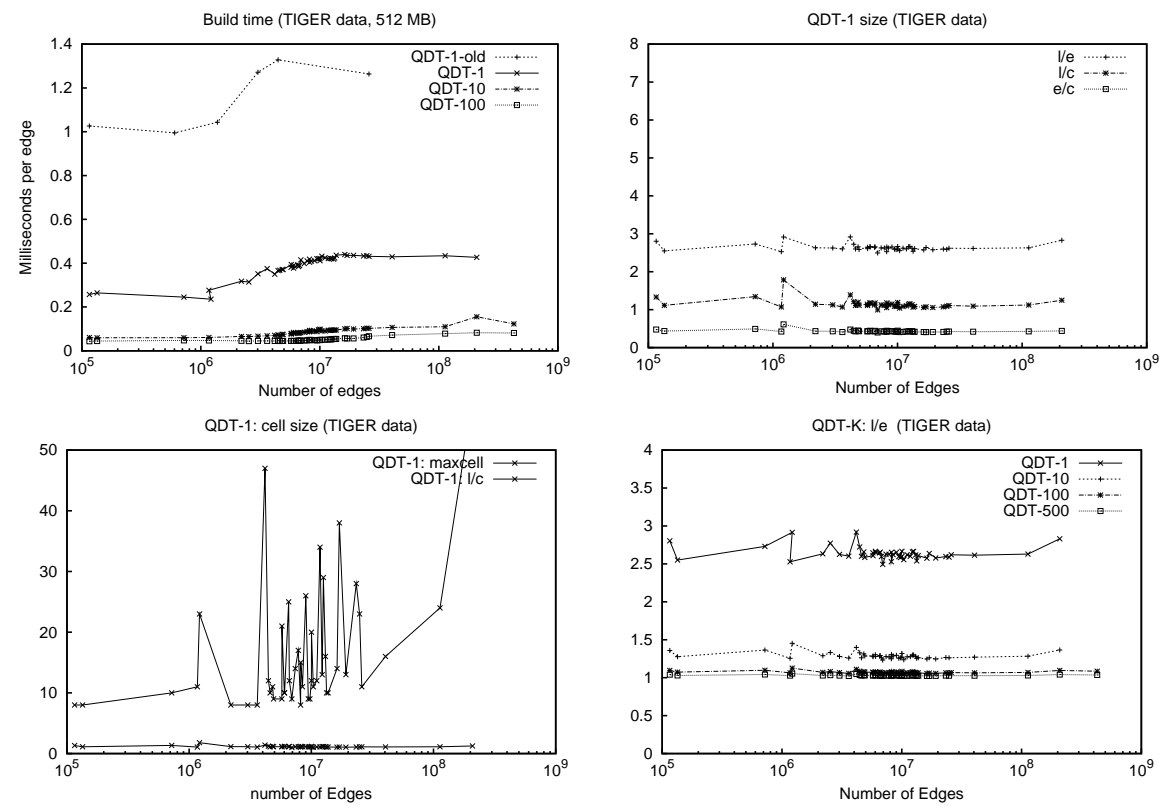

Fig. 3. Quadtree build times and sizes on TIGER data (512MB RAM).

has fewer cells and an overall smaller size. Empirically, for $k=10,100,500,1000$, QDT-K has $l=1.5 e, 1.1 e, 1.04 e$ and $1.03 e$, respectively. Table 2 shows the quadtree sizes and build times for the EastHalf bundle $\left(e=208.3 \cdot 10^{6}\right)$.

Segment intersection using quadtrees. To test the efficiency of segment intersection using quadtrees, we ran a set of experiments using a TIN $\left(e=53.9 \cdot 10^{6}\right)$ stored as QDT-1, and the TIGER datasets stored as QDT-K, for various values of $k$. To force all datasets to cover the same area, we scaled them to the unit square; the resulting intersections are artificial, and we only use them for run time analysis.

Overall, computing the intersecting segments is fast and scalable. From Table 3 we see that computing the overlay of 262 million edges can be done in under 1.8 hours if the quadtrees are given. Fig. 7 shows the times for $k=1,10,100,500$ and 1000. First, we note the big variations in time among the smaller TIGER sets. We suspect this is because the maximum cell size varies a lot (Fig. 3(c)), and overlay is sensitive to cell size (for each pair of cells that overlap, all edges in one are checked against all edges in the other). For the larger TIGER sets, we see two competing effects in the running time. On one hand, as $k$ increases, the size of a cell increases, and the time to compute the intersections between two cells increases. On the other hand, the overall number of cells and edge-cell intersections decrease, resulting in fewer cell-to-cell comparisons. The two effects, combined, cause the total time to first decrease as $k$ increases from 1 to 100 , and 
again increase for $k=500$. The optimal K-quadtree for segment intersection against QDT- 1 is not the one with $k=1$, as one might have expected, but seems to be one with $k \in[100,500]$.

\section{Conclusions}

We proposed a simple, I/O-efficient algorithm for the construction of a quadtree of a set of edges in the plane. For a user defined parameter $k \geq 1$, our quadtree has $O(n / k)$ cells with $O(k)$ vertices each, and can be built in $O(\operatorname{sort}(n+l))$ I/o's, where $l=O\left(n^{2} / k\right)$ is the total number of edge-cell intersections. The K-quadtree can trade off the size of a cell with the number of cells, overall size and construction time, and its I/O-efficient construction is simple and scalable. Our experiments confirm that K-quadtrees are viable for two classes of data used frequently in practice, TIN and TIGER. In our experiments we use test datasets of up to 427 million edges, two orders of magnitude larger than in related work $[7,6,8]$.

\section{References}

1. P. K. Agarwal, L. Arge, and A. Danner. From point cloud to grid DEM: a scalable approach. In Proc. 12th Symp. Spatial Data Handling (SDH 2006), pages 771-788.

2. A. Aggarwal and J. S. Vitter. The input/output complexity of sorting and related problems. Commun. ACM, 31:1116-1127, 1988.

3. L. Arge, R. D. Barve, D. Hutchinson, O. Procopiuc, L. Toma, J. Vahrenhold, D. E. Vengroff, and R. Wickremesinghe. TPIE user manual, 2005.

4. M. de Berg, H. Haverkort, S. Thite, and L. Toma. Star-quadtrees and guardquadtrees: I/O-efficient indexes for fat triangulations and low-density planar subdivisions. Computational Geometry, 43(5):493-513, 2010.

5. I. Gargantini. An effective way to represent quadtrees. Commun. ACM, 25(12):905-910, 1982.

6. G. Hjaltason and H. Samet. Improved bulk-loading algorithms for quadtrees. In Proc. ACM International Symposium on Advances in GIS, pages 110-115, 1999.

7. G. Hjaltason, H. Samet, and Y. Sussmann. Speeding up bulk-loading of quadtrees. In Proc. ACM International Symposium on Advances in GIS, 1997.

8. G. R. Hjaltason and H. Samet. Speeding up construction of PMR quadtree-based spatial indexes. VLDB Journal, 11:190-137, 2002.

9. E. Hoel and H. Samet. A qualitative comparison study of data structurers for large segment databases. In Proc. SIGMOD, pages 205-213, 1992.

10. R. Nelson and H. Samet. A population analysis for hierarchical data structures. In Proc. SIGMOD, pages 270-277, 1987.

11. H. Samet. Spatial Data Structures: Quadtrees, Octrees, and Other Hierarchical Methods. Addison-Wesley, Reading, MA, 1989.

12. H. Samet. Foundations of Multidimensional and Metric Data Structures. MorganKaufmann, 2006.

13. H. Samet, C. Shaffer, and R. Webber. The segment quadtree: a linear quadtreebased representation for linear features. Data Structures for Raster Graphics, pages 91-123, 1986. 
14. H. Samet and R. Webber. Storing a collection of polygons using quadtrees. $A C M$ Transactions on Graphics, 4(3):182-222, 1985.

15. L. Toma. External Memory Graph Algorithms and Applications to Geographic Information Systems. PhD thesis, Duke University, 2003. 


\section{Appendix}

\begin{tabular}{|l|r|r|r|}
\hline Dataset & $e$ & Max inc. & Min $L$ \\
\hline \hline Kaweah & $1.2 \cdot 10^{6}$ & 31 & .0704 \\
\hline Puerto Rico & $4.1 \cdot 10^{6}$ & 291 & .0010 \\
\hline Cumberlands & $5.1 \cdot 10^{6}$ & 44 & .0016 \\
\hline Sierra & $7.9 \cdot 10^{6}$ & 75 & .0137 \\
\hline Central App. & $10.1 \cdot 10^{6}$ & 62 & .0013 \\
\hline Hawaii & $19.7 \cdot 10^{6}$ & 356 & .0007 \\
\hline Haldem & $37.1 \cdot 10^{6}$ & 78 & .0097 \\
\hline Lower NE & $53.9 \cdot 10^{6}$ & 168 & .0021 \\
\hline
\end{tabular}

\begin{tabular}{|l|r|}
\hline Dataset & $e$ \\
\hline New England & $25.8 \cdot 10^{6}$ \\
\hline East Coast & $113.0 \cdot 10^{6}$ \\
\hline Eastern Half & $208.3 \cdot 10^{6}$ \\
\hline All USA & $427.7 \cdot 10^{6}$ \\
\hline
\end{tabular}

Fig. 4. (a) TIN datasets and their characteristics: the number of edges $e$, the maximum degree of a vertex, and the minimum angle (in degrees). (b) TIGER bundles sizes.

\begin{tabular}{|l|r|r|r|r|}
\hline & $c$ & $l$ & $l / c$ & build (minutes) \\
\hline \hline QDT-1-OLD & $32.5 \cdot 10^{6}$ & $158.8 \cdot 10^{6}$ & 4.8 & 1071 \\
\hline QDT-1 & $32.5 \cdot 10^{6}$ & $158.8 \cdot 10^{6}$ & 4.8 & 210 \\
\hline QDT-10 & $3.2 \cdot 10^{6}$ & $85.9 \cdot 10^{6}$ & 26.7 & 76 \\
\hline QDT-100 & $0.24 \cdot 10^{6}$ & $62.8 \cdot 10^{6}$ & 257.4 & 57 \\
\hline QDT-500 & $0.06 \cdot 10^{6}$ & $58.4 \cdot 10^{6}$ & 957.4 & 53 \\
\hline QDT-1000 & $0.02 \cdot 10^{6}$ & $56.5 \cdot 10^{6}$ & 2456.5 & 54 \\
\hline
\end{tabular}

Table 1. Quadtree size and build times on a test TIN dataset (LowerNE, $e=53.9 \cdot 10^{6}$ )

\begin{tabular}{|l|r|r|r|r|}
\hline & $c$ & $l$ & $l / c$ & build (minutes) \\
\hline \hline QDT-1 & $472.5 \cdot 10^{6}$ & $589.7 \cdot 10^{6}$ & 1.3 & 1,482 \\
\hline QDT-10 & $36.8 \cdot 10^{6}$ & $284.4 \cdot 10^{6}$ & 7.7 & 539 \\
\hline QDT-100 & $3.2 \cdot 10^{6}$ & $228.4 \cdot 10^{6}$ & 71.4 & 287 \\
\hline QDT-500 & $0.6 \cdot 10^{6}$ & $216.8 \cdot 10^{6}$ & 361.3 & 273 \\
\hline QDT-1000 & $0.3 \cdot 10^{6}$ & $214.2 \cdot 10^{6}$ & 714.0 & 280 \\
\hline
\end{tabular}

Table 2. Quadtree size and build times on a test TIGER dataset (EastHalf, $e=$ $208 \cdot 10^{6}$ ). 

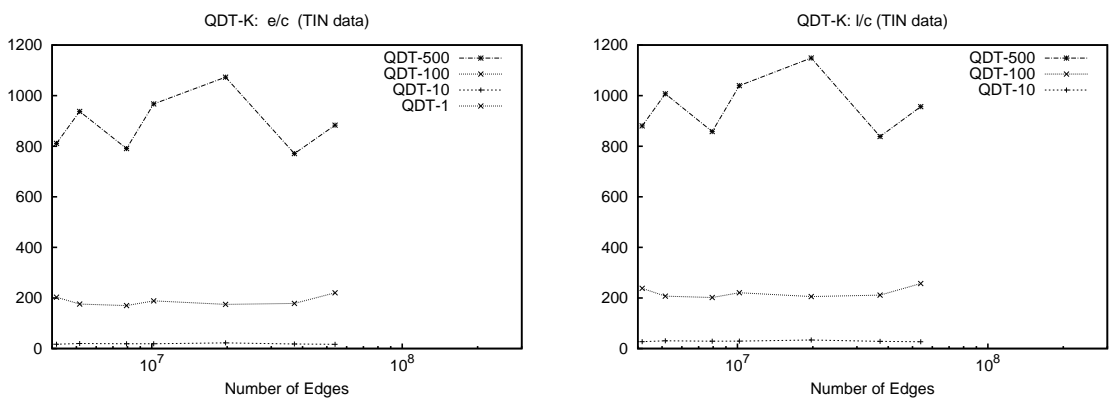

Fig. 5. K-quadtrees sizes on TIN data (512MB RAM).
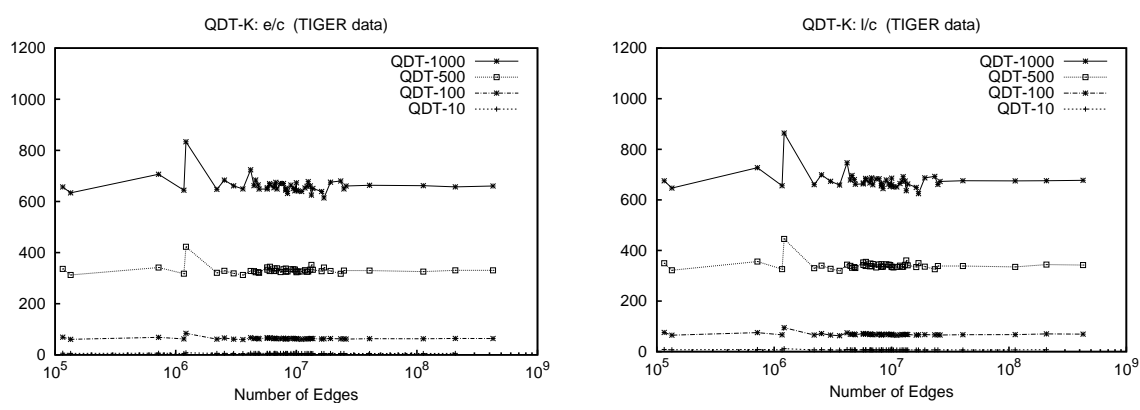

Fig. 6. K-quadtrees sizes on TIGER data (512MB RAM).

\begin{tabular}{|l|r|r|}
\hline & $l_{1}+l_{2}$ & time $(\mathrm{hr})$ \\
\hline \hline QDT-1 & $748.5 \cdot 10^{6}$ & 4.5 \\
\hline QDT-10 & $443.2 \cdot 10^{6}$ & 2.3 \\
\hline QDT-100 & $387.2 \cdot 10^{6}$ & 1.8 \\
\hline QDT-500 & $375.6 \cdot 10^{6}$ & 2.7 \\
\hline QDT-1000 & $373.1 \cdot 10^{6}$ & 4.0 \\
\hline
\end{tabular}

Table 3. Segment intersection between QDT-1 (LowerNE, $e=53.6 \cdot 10^{6}$ ) and QDT-K (EastHalf, $e=208 \cdot 10^{6}$ ), $512 \mathrm{MB}$ RAM.

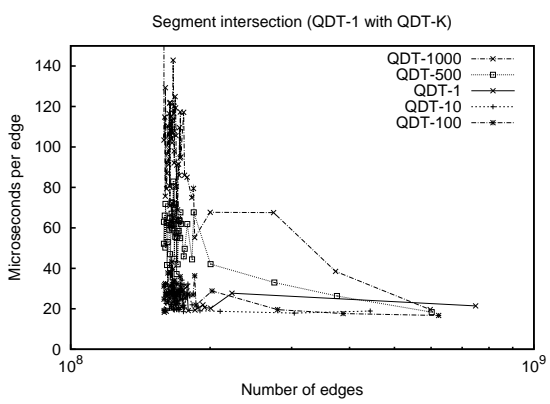

Fig. 7. Segment intersection (overlay) using quadtrees. 